\title{
Characterization of Oxidized and Fragmented Superoxide Dismutase Recruited on Liposome Surface
}

\author{
Le Quoc Tuan, Hiroshi Umakoshi, Toshinori Shimanouchi, and Ryoichi Kuboi*
}

\author{
Department of Chemical Science and Engineering, \\ Graduate School of Engineering Science, Osaka University \\ 1-3 Machikaneyama-cho, Toyonaka, Osaka 560-8531, Japan
}

The interaction of oxidized and fragmented SOD with liposomes has been reported to induce the original SOD-like enzymatic activity (L.Q. Tuan et al., Langmuir, 24 (2008) 350-354). The effect of several kinds of liposomes on their recruited activity of oxidized and fragmented SOD was first investigated. The addition of zwitterionic liposomes with high membrane fluidity or that with positive charge was found to increase the SOD-like activity of fragmented SOD although the negatively charged liposome has no effect on its activity. The SOD-like activity was found to be related to the adsorbed amount of the peptides on the liposome surface. The analyzed characteristics of the peptide, together with the above findings, imply that the liposome-recruited activity of the fragmented SOD was related with the recognition of the SOD fragment by the liposome caused by the combination of electrostatic and hydrophobic interaction, and hydrogen bonding between the peptide and liposome membrane.

Key words : membrane stress biotechnology / liposome / LIPOzyme / superoxide dismutase

\section{Introduction}

Superoxide dismutase (SOD) is a metalloenzyme that contains copper and zinc at its active site and catalyzes the dismutation of superoxide into oxygen and hydrogen peroxide ${ }^{1}$. However, under oxidative stress conditions, SOD fragments into small debris ${ }^{2,3)}$, which has no enzyme activity ${ }^{4 \sim 6}$ ). The inactivation of SOD is halted by some substrates ${ }^{6,7)}$ and by assisting with POPC liposomes ${ }^{8)}$.

It has recently reported that the liposome membrane can recruit the oxidized SOD fragment on its surface to produce a complex that has enzyme activity ${ }^{9}$. Among the fragments generated after SOD oxidation ${ }^{10,11)}$, some specific peptides contain its active site $(\sim 5$ and $\sim 10 \mathrm{kDa})^{12)}$, although these fragments have no SODlike activity. The recruiting mechanism of fragmented SOD on membrane was considered to involve the refolding and conformation of peptides by hydrophobic, electrostatic interaction, as well as mirodomain formation, in which liposomes perform a molecular chap-

* Corresponding Author

Tel \& Fax : +81-(0) 6-6850-6285

E-mail : kuboi@cheng.es.osaka-u.ac.jp erone-like function ${ }^{13 \sim 15)}$.

Much effort has been devoted to the design and development of artificial antioxidative enzymes ${ }^{16,17)}$. A model biomembrane, liposome, is a possible candidate to provide a common platform for different catalytic centers of SOD. Such a liposome herewith possesses several benefits in the regulation of catalytic activity, where it can provide (i) a nano hydrophobic environment, (ii) a stress-responsible character, (iii) a microdomain structure, and (iv) membrane-membrane interactions. Some researchers have reported the effectiveness of the use of a model biomembrane (liposome) as a platform to immobilize the functional catalytic center ${ }^{18 \sim 20)}$. Enzyme-like activity, such as that of SOD ${ }^{8,9,18)}$ and cholesterol oxidase ${ }^{21)}$, has already been regulated by the membrane properties of the liposome, as well as affording functional elements on the liposome surface. The above enzyme-like function of liposome, which can herewith be defined as "LIPOzyme" ${ }^{22)}$, can be utilized for the design of the artificial enzymes.

The final purpose is to characterize the oxidized and fragmented superoxide dismutase recruited on liposome surface, representing the SOD-like enzymatic activity. The relation between the enzymatic activity of 
recruited SOD and the charge and fluidity of membranes was first investigated. The adsorption of fragmented peptide on the membrane and SOD-like activity was observed. Based on characteristics of the fragment, a mechanism on the interaction of fragmented and oxidized SOD with various liposomes was finally investigated.

\section{Material and Method}

\subsection{Materials}

1,2-Dipalmitoyl-sn-glycero-3-phosphocholine (DPPC : $T_{\mathrm{m}}=42^{\circ} \mathrm{C}$ ), 1,2-dimyristoyl-sn-glycero- 3- phosphocholine (DMPC : $T_{\mathrm{m}}=23{ }^{\circ} \mathrm{C}$ ), 1-palmitoyl-2-oleoyl-snglycero-3-[phospho-rac-(1-glycerol)] (Sodium Salt) (POPG), 1,2-dioleoyl-3-trimethylammonium-Propane (Chloride Salt) (DOTAP) were purchased from Avanti Polar Lipids (Alabaster, AL, USA). Bovine erythrocyte $\mathrm{Cu}, \mathrm{Zn}-\mathrm{SOD}$ (EC 1.15.1.1), purchased from SigmaAldrich (St. Louis, MO, USA) with a specific activity of $4470 \mathrm{U} / \mathrm{mg}$ (product no. S2515-30KU, lot no. 125K740), was used without further purification. 1-Palmitoyl-2oleoyl-sn-glycero-3-phosphocholine (POPC) was purchased from Avanti Polar Lipids (Alabaster, AL, USA) and was used for liposome preparation. All other reagents of analytical grade were purchased from Wako Pure Chemical (Osaka, Japan).

\subsection{Preparations of Liposomes}

The lipids were dissolved in chloroform/methanol (10 $\mathrm{mM}$ lipid prepared will be diluted to $2 \mathrm{mM}$ for each experiment). POPC/Chl 28\% was composed form POPC and Cholesterol with $28 \%$ mole in comparison to POPC. After the solvent was evaporated, the resulting thin film was dried for at least 2 hours under a vacuum. The lipid film was hydrated by $50 \mathrm{mM}$ potassium phosphate buffer to form the multilamellar vesicles. The solution of the multilamellar vesicle was frozen in dry ice-ethanol $\left(-80^{\circ} \mathrm{C}\right)$ and incubated in the water bath above the phase-transition temperature. The above freezing-thawing treatment was repeated five times and was then passed through two stacked polycarbonate filters of $100 \mathrm{~nm}$ pore size by using an extrusion device to adjust the liposome size.

\subsection{Characterization of Membrane Fluidity}

The membrane fluidity of liposomes was determined by using a hydrophobic fluorescence probe, diphenyl hexatriene (DPH) ${ }^{23)}$. DPH was added to the solution of liposome-copolymers or liposome-proteins at a final concentration of $1 \mu \mathrm{M}$. The fluorescence polarization of the DPH probe in the liposome membrane was measured at a wavelength of $360 \mathrm{~nm}$ for the excitation and $430 \mathrm{~nm}$ for the emissions. The fluorescence intensity of the DPH was measured by using the fluorescent spectrophotometer (JASCO FP-777, Japan). The degree of fluorescence polarization $(P)$ was calculated using the following equation:

$$
\frac{1}{P}=\frac{\left(I_{/ /}+I_{\perp}\right)}{\left(I_{/ /}+I_{\perp}\right)}
$$

where $I_{/ /}$and $I_{\perp}$ are the intensities of the light emitted with its polarization plane parallel $(/ /)$ and perpendicular $(\perp)$ to that of the exciting beam, respectively. The term "fluidity" is inversely proportional to the degree of fluorescence polarization of the probe; that is, the "membrane fluidity" of the interior of the membrane was defined by $(1 / P)$ of $\mathrm{DPH}$.

\subsection{Analytical Methods}

Analysis of $\mathrm{Cu}, \mathrm{Zn}-\mathrm{SOD}$ fragmentation by $\mathrm{H}_{2} \mathrm{O}_{2}$. Cu, Zn-SOD $(2 \mu \mathrm{M})$ was incubated with $\mathrm{H}_{2} \mathrm{O}_{2}(2 \mathrm{mM})$ in phosphate buffer ( $\mathrm{pH} 7.4$ ) at $37^{\circ} \mathrm{C}$ for $12 \mathrm{~h}$. The enzymatic activity and protein concentration of fragmented SOD were determined after the incubation of SOD with $\mathrm{H}_{2} \mathrm{O}_{2}$. The SDS-PAGE technique was used to analyze SOD fragmentation.

For the SOD activity, a highly water-soluble tretrazolium salt, WST-1 [2-(4-lopophenyl)-3-(4-nitrophenyl)5-(2,4-disulfophenyl)-2H-tetrazolium, monosodium salt], produces a water-soluble formazan dye upon reduction with a superoxide anion, where the rate of the reduction with $\mathrm{O}_{2}{ }^{-}$is linearly related to the xanthine oxidase (XO) activity and is inhibited by SOD. The absorption spectrum of WST-1 formazan was measured at $450 \mathrm{~nm}$, and the SOD activity as an inhibition activity can be quantified through the decrease in color development ${ }^{24)}$. Standard SOD used with the concentration of $2 \mu \mathrm{M}$ displays an enzymatic activity $100 \%$. Standard SOD converts total superoxide anion $\left(\mathrm{O}_{2}{ }^{-}\right)$into hydrogen peroxide and molecular oxygen, thus, inhibits $100 \%$ the formation of WST-1 formazan from WST-1 by superoxide anion.

The BCA Protein Assay Kit was used to determine the protein concentrations. The protein was precipitat- 
ed in a cold acetone solution to separate it from contaminants, so that a more accurate estimation of protein content in the sample could be obtained. The mixture was then centrifuged at $15,000 \mathrm{rpm}$ for $20 \mathrm{~min}$. Pellets solubilized in $50 \mu 1 \mathrm{H}_{2} \mathrm{O}$ were added to $1000 \mu \mathrm{l}$ of BCA reagent solution and incubated for $30 \mathrm{~min}$ at $37^{\circ} \mathrm{C}$, after which the absorbance at $562 \mathrm{~nm}$ was measured. A standard curve was set up to analyze the protein concentrations ${ }^{25)}$.

Ultrafiltration of $\mathrm{H}_{2} \mathrm{O}_{2}$-Treated SOD. Ultrafiltration using an Ultrafree-MC filter (Millipore, Billerica, MA, USA) with a molecular mass cutoff of $10 \mathrm{kDa}$ was applied for fragmentation analysis. SOD, after being treated by $\mathrm{H}_{2} \mathrm{O}_{2}$, was applied to an ultrafiltration tube and centrifuged at $15,000 \mathrm{rpm}$ for $30 \mathrm{~min}$ at room temperature. Aliquots were then analyzed by reversephase HPLC, and their enzymatic activity was determined. The filter with a molecular mass cutoff of 3 $\mathrm{kDa}$ was applied to remove free copper released from active site.

For the reverse-phase HPLC, a Shimadzu (Kyoto, Japan) HPLC system equipped with an FCV-10AL pump, a DGU-20A3 degasser, an SPD-10A UV-VS detector, and an LC-10AD liquid chromatograph was used. Elution profiles were monitored at $220 \mathrm{~nm}$ on the UV detector. The mobile phase of acetonitrile/water (v/v 7/3) with a flow rate of $1 \mathrm{~mL} / \mathrm{min}$ was applied at $25{ }^{\circ}$. An STR ODS-M column $(0.46 \mathrm{~cm} \times 15 \mathrm{~cm})$, in which the particle surface was octadecylated, was used throughout this study.

Treatment of SOD and fragmented SOD. The SOD, totally oxidized into fragments for $12 \mathrm{~h}$ in $2 \mathrm{mM} \mathrm{H}_{2} \mathrm{O}_{2}$, was ultrafiltered by a $10 \mathrm{kDa}$ molecular cutoff filter to obtain the potentially active debris (fragmented SOD) containing the active sites ${ }^{9,12)}$. The $10 \mathrm{kDa}$ fragments filtrated from ultrafiltration were incubated with POPC $2 \mathrm{mM}$ and metal ions $\left(\mathrm{Cu}^{2+}\right.$ and $\left.\mathrm{Zn}^{2+}\right) 10 \mu \mathrm{M}$ for each of several alternatives of POPC or metal ions in phosphate buffer at $37^{\circ} \mathrm{C}$ for $12 \mathrm{~h}$. The enzymatic activity of the complex of liposomes, fragmented SOD, and metal ions was determined. Amino acid sequences were analyzed by Protein Institute.

Statistical Analysis. Results are expressed as means \pm standard deviation (SD). All experiments were performed at least in triplicate. Data distribution was analyzed, and statistical differences were evaluated using Student's t-test. A P value of $<0.05 \%$ was considered significant.

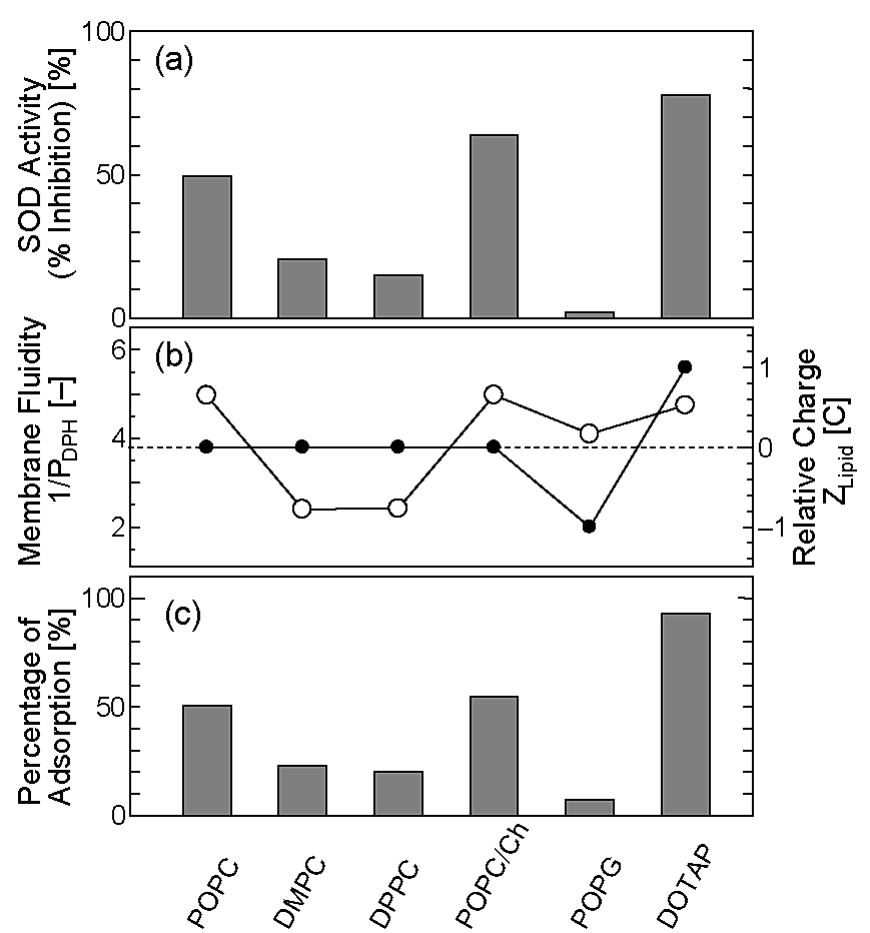

Fig. 1 Effect of Liposome Type on Recovered Activity of Fr.-SOD and Its Adsorption on the Liposome. (a) Recovered Fr.-SOD $(2 \mu \mathrm{M})$ was treated with $10 \mathrm{mM}$ $\mathrm{Cu}^{2+}$ and $\mathrm{Zn}^{2+}$ with various liposomes (2 mM lipid concentration). The SOD activity was shown as a percentage of inhibitory activity. (b) The basic characteristics of liposome membrane, such as membrane fluidity measured by DPH $(O)$ and relative charge of the hydrophilic head group of the lipid molecules (O). (c) The percentage of the adsorption of Fr.-SOD on the surface of liposome. After mixing the Fr.-SOD and liposome, the non-adsorbed Fr.-SOD was recovered by ultrafiltration. The percentage of adsorption was determined from the mass-balance of the protein concentration before and after the ultrafiltration.

\section{Results and Discussion}

\subsection{Activity and Adsorption of Oxidized and Fragmented SOD Recruited on Various Liposomes}

The liposome has reported to recruit the oxidized and fragmented SOD on its surface and induce the SOD-like activity ${ }^{9}$. The fragmented SOD was also shown to acquire its secondary structure through the coordination with metal ions on the surface of the liposome ${ }^{26)}$. The effect of liposome types on the liposomerecruited activity was investigated. 

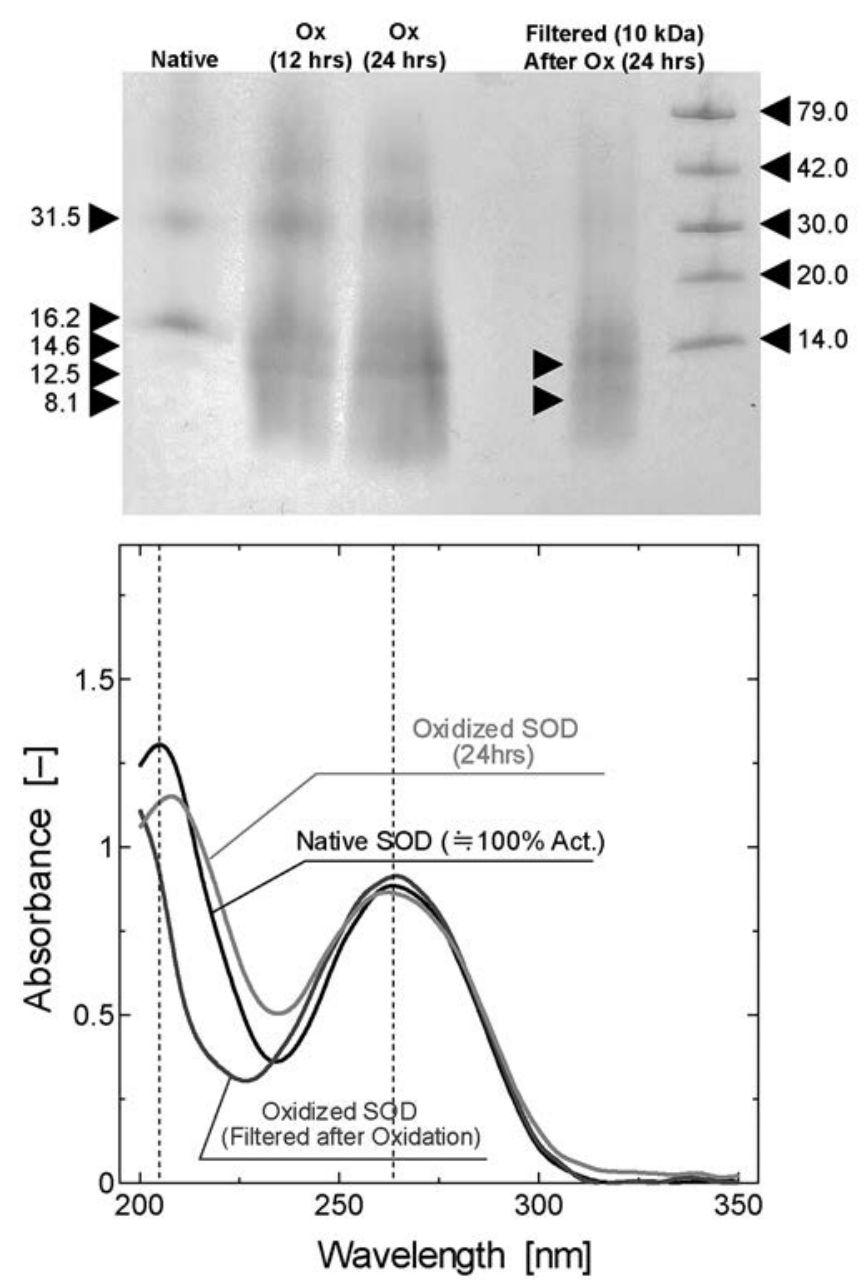

Fig. 2 Basic Characteristics of Fr.-SOD. (a) The CBBstained image of SDS-PAGE analyzed gel. The native SOD, oxidized SOD (12 and 24 hours) and recovered SOD were applied for the SDS-PAGE analysis. (b) The UV spectra of the native SOD, oxidized SOD and the recovered SOD.

Fig. 1(a) shows the SOD-like activity of the fragmented SOD $(2 \mu \mathrm{M})$ with $\mathrm{Cu}$ and $\mathrm{Zn}(10 \mathrm{mM})$ in the presence of various types of liposomes ( $2 \mathrm{mM}$ lipid). In the presence of POPC liposomes, the SOD activity was approximately $50 \%$, which is compatible with the previous results ${ }^{9,26)}$. The value was reduced depending on the type of zwitterionic lipid (DMPC and DPPC). As shown in Fig. 1(b), the membrane fluidity was reduced in correspondence with the decrease of the SOD activity. It has been reported that the phase transition temperature of the POPC, DMPC, and DPPC liposomes are $-4{ }^{\circ} \mathrm{C}, 23{ }^{\circ} \mathrm{C}$, and $45^{\circ} \mathrm{C}$, respectively ${ }^{27}$, where the POPC liposomes exists in a liquid crystalline phase at the temperature tested here. It has also reported that the membrane fluidity of the liposome could increase the hydrophobicity of the liposome surface ${ }^{28,29)}$. The above tendency of the SOD activity could be related to the hydrophobic interaction between the fragmented peptides and liposome membrane.

As shown in Fig. 1(a), the SOD activity was further increased in the case of the POPC/Ch liposome, resulting in the $62 \%$ activity, although the membrane fluidity was not significantly increased (Fig. 1(b)). It has been reported that the $\mathrm{POPC} / \mathrm{Ch}$ liposomes possess microdomain structures on its surface and the microdomain structure shows higher affinity with the peptide components ${ }^{30)}$ owing to the hydrogen bond stabilization of the peptide on the membrane ${ }^{21}$. It has been reported that the stability of the hydrogen bonds of the main backbone of the peptide could be modulated with the wrapping hydrophobic residues ${ }^{31)}$. The hydrogen bonds, which contribute to the conformational change of fragment and its interaction with liposome, could be enhanced in the hydrophobic environment provided by the liposome membrane.

The effect of the charged liposome was also investigated by adding the POPG and DOTAP liposome. An opposite effect on the liposome addition was obtained. The SOD-like enzymatic activity was reached to $78 \%$ in the presence of DOTAP while the value was only $3 \%$ in the case of POPG liposomes (right keys in Fig. 1(a)). The calculated net charge of head group of a lipid molecule at this experimental condition was shown in Fig. 1(b). Different from the above liposomes (POPC, DMPC, DPPC, and POPC/Ch), the surface of POPG liposomes has been negatively charged owing to the phosphate group and that of DOTAP has been positively charged by the contribution of the tetra-ammonium group. As can be seen in the chemical structure of the PC-lipids, it also has a positively charged choline-group together with the phosphate group, implying that the edge of membrane surface has positively-charged group. It has been reported that the liposome could interact with the negatively charged biomacromolecule (i.e. RNA) because of their electrostatic interaction ${ }^{32}$. The above results and the previous findings show that the fragmented SOD to be recruited on the surface of liposome because of the electrostatic interaction between them.

In all the condition of various liposomes, the adsorption amounts of the fragmented SOD on the liposome were analyzed by using the ultrafiltration technique as shown in Fig. 1(c). The percentage of the adsorption was well related to the variation of the SOD-like activi- 


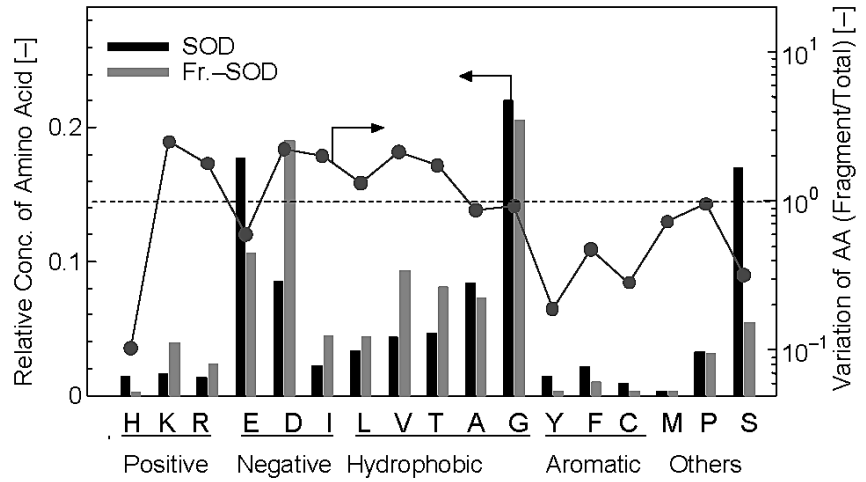

Fig. 3 Relative Concentration of Amino Acids of Native SOD and Fr.-SOD. The amino acid composition was determined by reverse-phase HPLC after the hydrolysis of the peptides in $\mathrm{NaOH}$ solution (Analysis in Protein Institute: See experimental Section). The variation of relative concentration of each amino acid was shown as the relative ratio of the amino acid concentration of Fr.-SOD against the native SOD.

ty. The adsorption behaviors of the SOD fragment show that the "liposome-recruited activity of the oxidized and fragmented SOD" could be derived from the interaction (recognition) of the fragmented peptides with the liposome surface, implying the interaction could be governed by the electrostatic interaction, hydrophobic interaction and hydrogen bonds and the fragment to be recruited on the liposome surface could have a negative charge, hydrophobic surface and unstable hydrogen bonds not wrapped by hydrophobic environment. However, the recruited SOD-like activity was not always followed this phenomenon. For example, the interaction of oxidized POPC and fragmented SOD was also investigated (data not shown). In this condition, the absorption of fragmented SOD to membrane was so high although the recruited SOD-like activity was low. The above results imply that there could be, at least, two steps such as (i) peptide absorption and (ii) reconstruction of its conformation to induce the liposome-recruited enzymatic activity. A mechanism of the above liposome function is now under investigation.

\subsection{Characteristics of Oxidized and Frag- mented Peptide Recruited on Liposome Surface}

Based on the above results, the oxidized and fragmented peptide recruited on the liposome surface was furthermore characterized.
The molecular weight of the oxidized and fragmented SOD was analyzed by using the SDS-PAGE. Fig. 2(a) shows the CBB-stained image of the analyzed gel. In the case of the native SOD, the dimer (31.5 $\mathrm{kDa})$ and monomer (16.2 kDa) were observed (Lane 1). By treating the SOD with $2 \mathrm{mM} \mathrm{H}_{2} \mathrm{O}_{2}$, such original bands were gradually lost and small fragments were newly formed (Lane 2 and 3). The liposome recruited fragment recovered finally shows two main bands at $12.5 \mathrm{kDa}$ and $8.1 \mathrm{kDa}$ and also show much small fragments below these bands (Lane 4). The molecular weight of the finally recovered fragments could range from $8 \mathrm{kDa}$ to $10 \mathrm{kDa}$. The $\mathrm{UV}$ spectra of the recovered SOD were contrasted with the native one as shown in Fig. 2(b). Similarly in the case of the native SOD, a strong peak was observed at $260 \mathrm{~nm}$, showing that the SOD fragment could possess the His and aromatic groups in its structure. The comparison of the absorbance at $205 \mathrm{~nm}$ shows that the SOD fragment could be abundant in the amino acids harboring the carboxyl groups as compared with the simply-oxidized fragment. The amino acid composition of the native SOD and the fragmented SOD was further investigated. Fig. 3 shows the relative concentration of the 18 amino acids of native SOD (black keys) and the fragmented SOD (red keys). The variation of amino acid of fragmented SOD against the native one was also shown as a relative ratio of these values. Although the decrease in the relative concentration of His residue was observed, the increase of the charged amino acids and hydrophobic amino acids was observed. The above results show that the fragmented peptide has a charged and hydrophobic surface and also possess the His residues although its absolute amounts were lower than those of the original one.

The surface characteristic of the fragmented SOD was finally discussed based on the above results and also the basic database of the native SOD. The structure data of the $\mathrm{Cu} / \mathrm{Zn}-\mathrm{SOD}$ (PDB ID : $1 \mathrm{CBJ}$ ) were used for the discussion in Fig. 4. Fig. 4(a) shows the position of the His residues in the SOD structure, where the 6 His residues contribute to the formation of its activity center among 8 residues. The position of the secondary structure of SOD was shown as solid lines in Fig. 4(b), where $\beta$-sheet structure is mainly distributed along them except for the $\alpha$-helix structure neighboring to its activity center. The relative score on the secondary structures, predicted by Chou and 


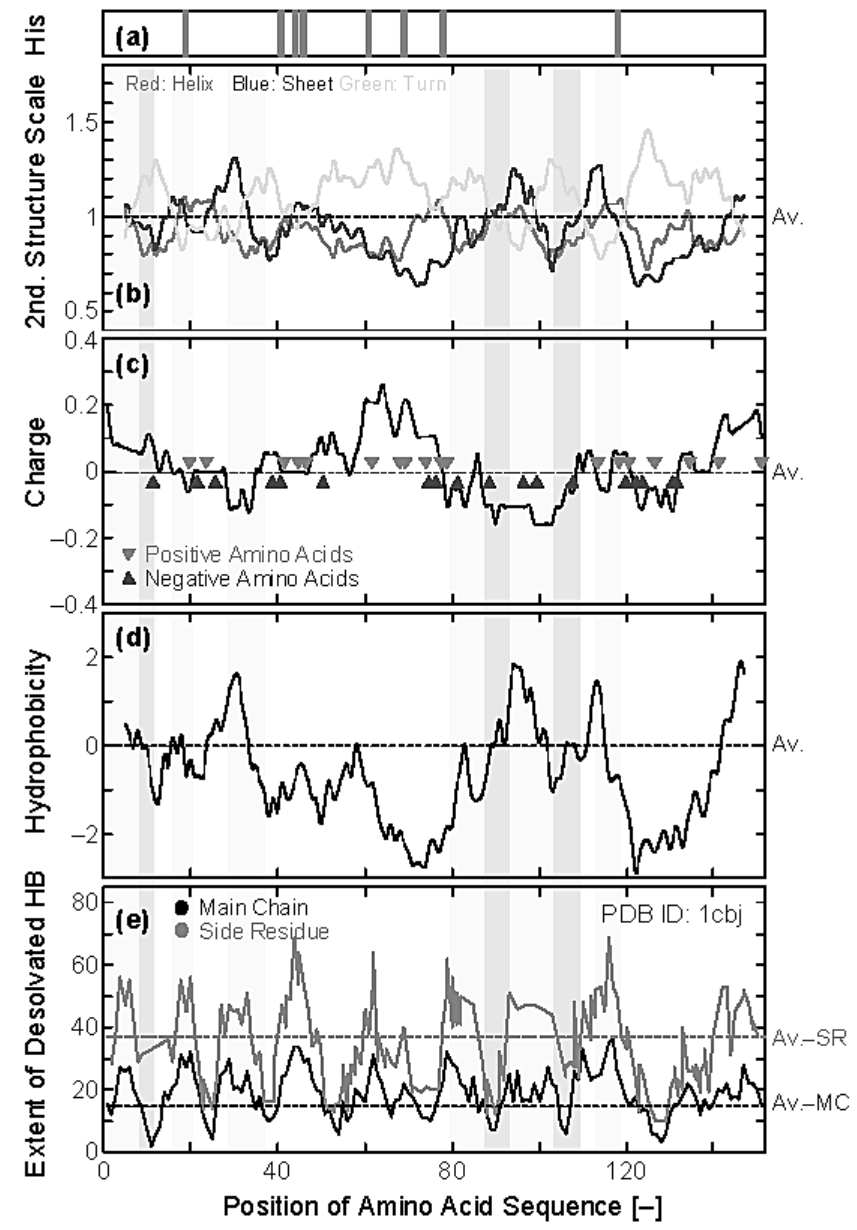

Fasman method, was also shown as curves in Fig. 4(b). Although the predicted score in general matched with the actual secondary structure in the case of $\beta$-sheet or $\beta$-turn, some unmatched domains were observed in focusing on the predicted $\alpha$-helix value; where the relative scale was high in Nos.18-30, 74-80, 95-100, 92-124 although the actual structure was $\beta$-sheet or $\beta$-turn structure. Fig. 4(c) shows the relative values of the surface charge of the side residues of the amino acids, where the negative charge were clustered in the N-terminal region (No. 10-40) and middle region (No. 7090). The hydropathy plot was shown in Fig. 4(d), where the hydrophobic residues were assembled in some domains along the amino acid sequence (Nos.112, 22-38, 81-120, and 130-152). As shown in Fig. 4(e), the stability of the hydrogen bonds of the main chain and side residue were also plotted based on the previous method on the calculation of the hydrogen bondwrapping ${ }^{31,33)}$. There are some valleys in the curve of hydrogen bond stability of both main chain and side residues. The results obtained in the previous section show that the SOD fragment has negative charge and
Fig. 4 Basic Characteristics of SOD at Any Position along Amino Acid Sequence from N- to C-Terminal. All the data were analyzed based on structure data of domain A of native SOD registered in Protein Data Bank (http://www.rcsb.org/, PDB ID: 1CBJ). (a) The position of His and (b) secondary structure (red: $\alpha$-helix, blue: $\beta$-sheet, green: $\beta$-turn: Curve; prediction by Chou and Fasman Method; Bold Line in the top; actual secondary structure). (c) The moving-average of the surface net charge of neighboring nine amino acids by assuming the Glu and Asp show -1 and His, Arg, and Lys show +1 . (d) The movingaverage of the surface net charge of neighboring nine amino acids by using the hydrophobicity scale reported previously (Kyte J. and Doolittle R.F., J. Mol. Biol., 157, 105 (1982)). (e) The extents of desolvation of hydrogen bond by hydrophobic residues in main chain and side residue of SOD, where the hydrogen bonds at any position are stabilized in the case of the higher extents of desolvated hydrogen bond (Fernandez, A. amd Berry, R.S., PNAS, 100, 2391 (2003) / Fernandez, A. amd Scott, R., Biophys. J., 85, 1914 (2003)). Yellow-colored bands in (b)-(e) shows the domain with both negative charge and high hydrophobicity and blue-colored bands shows that with low stability of hydrogen bonds among the yellow regions.

hydrophobic surface (Fig. 1). Among the possible sequences of the SOD, the amino aside residues harboring the above two characteristics were picked up and were shown as yellow-colored bands. It has been reported that the proteins with under-wrapped hydrogen bonds could favorably interact with the lipid membrane ${ }^{31}$. Among the yellow-colored bands, the specific domains with low stability of the hydrogen bond were further shown as blue-colored bands.

Although the fragment recruited on the liposome has not been specified yet, it is suggested that the parts of the SOD shown as yellow- and blue-colored bands in Fig. 4 could be candidates of its fragment considering the data on the SOD-like activity recruited on the various types of liposomes. Liposome membranes that can recruit the SOD fragment could have potentials to recognize the peptide with unstable hydrogen-bond (Fig. 4(e)), considering the potential ability to form the $\alpha$-helix of the peptide (unmatched region of predicted and practical secondary structure in Fig. 4(b)). The above results also imply that the obtained fragment characteristics could provide useful information on the 
"molecular-recognition function" of the lipid membrane itself.

\section{Acknowledgement}

The fundamental concept of this study was supported by the Research Group of "Membrane Stress Biotechnology". It was partly supported by a Grant-inAid for Scientific Research (No. 15206089, 16686046, 16760635, 17656268, 19656203, 19656220, and 20360350) from the Ministry of Education, Science, Sports, and Culture of Japan, a grant from the 21st Century COE program "Creation of Integrated EcoChemistry" and the Global COE program "BioEnvironmental Chemistry" of the Japan Society for the Promotion of Science (JSPS). The authors are grateful to the Research Center for Solar Energy Chemistry of Osaka University and the Gas hydrate Analyzing System of Osaka University. The authors acknowledge Prof. Dr. Ariel Fernandez (Rice University) for his kind advice on the calculation of extent of desolvated hydrogen bond. L.Q. Tuan acknowledges financial support from the Ministry of Education and Training, Vietnam (MOET).

\section{Refferences}

1) McCord JM, Fridovich IJ : Biol. Chem., 244, 6049-6055 (1969)

2) Choi SY, Kwon HY, Kwon OB, Kang JW : Biochim. Biophys. Acta, 1472, 651-657 (1999)

3) Kang JH, Kim SM : Mol. Cell, 7, 553-558 (1997)

4) Salo DC, Pacifici RE, Lin SW, Giulivi C, Davies KJA : J. Biol. Chem., 265, 11919-11927 (1990)

5) Uchida K, Kawakishi SJ : Biol. Chem., 269, 2405-2410 (1994)

6) Goldstone AB, Liochev SI, Fridovich I : Free Radical Biol. Med., 41, 1860-1863 (2006)

7) Kang JH, Kim KS, Choi SY, Kwon HY, Won MH, Kang TC : Biochim. Biophys. Acta, 1570 , 89-96 (2002)

8) Nagami H, Yoshimoto N, Umakoshi H, Shimanouchi T, Kuboi R: J. Biosci. Bioeng., 99, 423-428 (2005)

9) Tuan LQ, Umakoshi H, Shimanouchi T, Kuboi R : Langmuir, 24, 350-354 (2008).

10) Ookawara $\mathrm{T}$, Kawamura $N$, Kitagawas $Y$, Taniguchi $N: J$. Biol. Chem., 267, 18505-18510 (1992)

11) Kurahashi T, Miyazaki A, Suwan $\mathrm{S}$, Isobe $\mathrm{M}: J . A m$. Chem. Soc., 123, 9268-9278 (2001)

12) Ramirez DC, Mejiba SEG, Mason RP : Free Radical Biol. Med., 38, 201-214 (2005)

13) Yoshimoto M, Kuboi R : Biotechnol. Prog., 15, 480-487 (1999)

14) Yoshimoto M, Shimanouchi T, Umakoshi H, Kuboi R: J.
Chromatogr. B, 743, 93-99 (2000)

15) Kuboi K, Mawatari T, Yoshimoto M : J. Biosci. Bioeng., 90, 14-19 (2000)

16) Kirby AJ : Angew. Chem. Int'l Ed., 35, 707-724 (1996)

17) Batinic-Harber I, Benov L, Spasojevic I, Fridovich I : J. Biol. Chem., 273, 24521-24528 (1996)

18) Nagami H, Umakoshi H, Shimanouchi T, Kuboi R : Biochem. Eng. J., 21, 221-227 (2004)

19) Walde P : Origins of Life and Evolution of Biosphere, 36, 109-150 (2006)

20) Murakami Y, Kikuchi J, Hisaeda Y, Hayashida O : Chem. Rev., 96, 721-758 (1996)

21) Yoshimoto N, Tasaki M, Shimanouchi T, Umakoshi $H$, Kuboi R: J. Biosci. Bioeng., 100, 455-459 (2005)

22) Kuboi R, Umakoshi H, Shimanouchi T : Proc. of 1st European Chemistry Congress (Budapest Hungary, August 27-31), 2006, C-PO-78, p.50-51 (See Supplement Reference)

23) Nagami H, Umakoshi H, Shimanouchi T, Kuboi R, Baba Y : Solv. Extr. Res. Dev. Japan, 11, 159-165 (2004)

24) Peskin AV, Winterbourn CC : Clin. Chim. Acta, 293, 157$166(2000)$

25) Smith PK, Krohn RI, Hermanson GT, Mallia AK, Gartner FH, Provenzano MD, Fujimoto EK, Goeke NM, Olson BJ, Klenk DC : Anal. Biochem., 150, 76-85 (1985)

26) Tuan LQ, Umakoshi H, Shimanouchi T, Kuboi R : submitted (2008)

27) Umakoshi H, Morimoto $K$, Ohama $Y$, Nagami $H$, Shimanouchi T, Kuboi R : Langmuir, 24, 4451-4455 (2008)

28) Kuboi R, Yoshimoto M, Walde P, Luisi PL : Biotechnol. Prog., 13, 828-836 (1997)

29) Umakoshi H, Kuboi R : J. Chromatogr. B: Biomedi. Sci. Appl., 711, 217-222 (1998)

30) Taylor KMP, Roseman MA : Biochim. Biophys. Acta, 1278, 35-40 (1996)

31) Fernandez A, Berry RS : PNAS, 100, 2391-2396 (2003)

32) Janas, T, Janas, T, Yarus M : Nucleic Acids Research, 34, 2128-2136 (2006)

33) Fernandez A, Scotty R : Biophysical Journal, 85, 1914-1928 (2003)

(Received 12 May 2008; Accepted 21 June 2008) 